\title{
Treinamento em radiologia utilizando técnicas de realidade virtual: uma breve
}

\section{revisão do estado da arte}

\author{
Training in radiology using virtual reality techniques: a brief state of the art review \\ Formación en radiología utilizando técnicas de realidad virtual: una breve revisión del estado del
}

arte

Recebido: 29/10/2021 | Revisado: 07/11/2021 | Aceito: 10/11/2021 | Publicado: 14/11/2021

Paulo Camargos Silva

ORCID: https://orcid.org/0000-0002-3542-7368

Universidade Federal de Uberlândia, Brasil

E-mail: paulo.camargos@ufu.br

Gabriel Fernandes Cyrino

ORCID: https://orcid.org/0000-0001-7307-0171

Universidade Federal de Uberlândia, Brasil

E-mail: gabrielcyrino@ufu.br

Camilo de Lelis Barreto Junior

ORCID: https://orcid.org/0000-0001-7250-605X

Universidade Federal de Uberlândia, Brasil

E-mail: camilobarreto@ufu.br

Edgard Afonso Lamounier Junior

ORCID: https://orcid.org/0000-0001-6293-9521

Universidade Federal de Uberlândia, Brasil

E-mail: lamounier@ufu.br

Alexandre Cardoso

ORCID: https://orcid.org/0000-0002-2023-9647

Universidade Federal de Uberlândia, Brasil

E-mail: alexandre@ufu.br

Ana Claudia Patrocinio

ORCID: https://orcid.org/0000-0001-9376-7689

Universidade Federal de Uberlândia, Brasil

E-mail: ana.patrocinio@ufu.br

\begin{abstract}
Resumo
As áreas da radiologia diagnóstica e intervencionista utilizam amplamente imagens geradas através de raios X. Como exemplo, pode-se citar a mamografia como exame com base nessa modalidade de geração de imagens. O procedimento de aquisição de imagens é realizado por profissionais que devem ser qualificados para exercer a atividade, bem como devem passar por cursos de atualização e/ou reciclagem. Nesta via, treinamentos utilizando técnicas de Realidade Virtual tem sido amplamente utilizado na área da saúde. Assim, este trabalho apresenta uma breve revisão bibliográfica e o estado da arte de sistemas computacionais que utilizam a Realidade Virtual para treinar os profissionais em radiografia.
\end{abstract}

Palavras-chave: Estado da arte; Radiologia; Realidade virtual; Treinamento.

\begin{abstract}
The diagnostic and interventional radiology fields make extensive use of images generated through X-rays. As an example, mammography can be mentioned as an exam based on this modality of imaging. The image acquisition procedure is performed by professionals who must be qualified to carry out the activity, as well as undergo refresher and/or refresher courses. In this way, training using Virtual Reality techniques has been widely used in the health area. Thus, this work presents a brief bibliographical review and the state of the art of computational systems that use Virtual Reality to train professionals in radiography.
\end{abstract}

Keywords: State of art; Radiology; Virtual reality; Training.

\section{Resumen}

Las áreas de radiología diagnóstica e intervencionista hacen un uso extensivo de las imágenes generadas a través de los rayos X. Como ejemplo, se puede mencionar la mamografía como un examen basado en esta modalidad de imagen. El procedimiento de adquisición de imagen es realizado por profesionales que deben estar capacitados para realizar la actividad, así como quienes deben realizar cursos de actualización y / o actualización. De esta forma, la formación mediante técnicas de Realidad Virtual ha sido muy utilizada en el área de la salud. Así, este trabajo 
presenta una breve revisión bibliográfica y el estado del arte de los sistemas computacionales que utilizan la Realidad Virtual para formar profesionales en radiografía.

Palabras clave: Estado del arte; Radiología; Realidad virtual; Capacitación.

\section{Introdução}

A geração de imagens por raios X é essencial nos campos da radiologia diagnóstica e intervencionista. Na radiologia diagnóstica as imagens ajudam a identificar doenças por meio da visualização de estruturas internas do corpo. Na radiologia intervencionista, as imagens podem ser usadas para guiar e orientar procedimentos cirúrgicos. Como exemplo, pode-se citar exames de mamografia para detecção do câncer de mama, cuja geração de imagens por raios X é amplamente aplicada.

De acordo com a Organização Pan-Americana de Saúde (OPAS), o câncer é a segunda principal causa de morte no mundo, responsável por 9,6 milhões de mortes somente no ano de 2018. (OPAS, 2021). A Organização Mundial da Saúde (OMS) estima que ocorre mais de um milhão de novos casos anualmente no mundo. Entre as mulheres brasileiras o câncer de mama é o mais frequente (sem considerar o câncer de pele do tipo não-melanoma) e a segunda principal causa de morte (INCA, 2021).

Em um levantamento realizado no ano de 2019 pelo Instituto Nacional de Câncer José Alencar Gomes da Silva (INCA), foram estimados 198.840 novos casos durante o triênio 2020/2022, totalizando 66.280 casos para cada ano (INCA, 2019). A mamografia é um dos exames que utiliza raios X. Este exame é considerado o exame padrão no mundo para rastreamento da doença (Coop et al., 2016), sendo recomendado pelo ministério da saúde (INCA, 2015; Migowski et al., 2018) a ser realizado a cada 2 anos por mulheres entre 40 e 59 anos.

A qualidade da imagem gerada nos exames está diretamente relacionada à capacidade de detecção de uma anormalidade (Caldas et al., 2005). A garantia da qualidade envolve critérios técnicos como a aplicação de testes periódicos nos equipamentos e clínicos como avaliação de filmes, exposição e definição da imagem (Sabino et al., 2014).

Em um estudo, (Sabino et al., 2014) realizou a implantação de um programa de controle de qualidade em um hospital de referência. Durante a avaliação dos resultados do programa, foi constatado que $89 \%$ das falhas estavam relacionadas a um elemento específico do procedimento aplicado pelos técnicos em radiologia. Este estudo destaca o treinamento contínuo de profissionais como essencial para uma maior qualidade nos exames.

Além disso, a Resolução da Diretoria Colegiada (RDC) No 330 de 2019 estabelece os requisitos sanitários de estabelecimentos e serviços de radiologia diagnóstica, bem como regulamenta o controle de exposições ocupacionais e do público devido ao uso de tecnologias radiológicas. A RDC No 330 define, conforme o Artigo 5, que estabelecimento e serviços de radiologia diagnóstica e/ou de intervenção devem implementar programas de garantia de qualidade, educação permanente e proteção radiológico e realizem o controle sobre tais treinamentos (Agência Nacional de Vigilância Sanitária, 2019). A Realidade Virtual (RV) vem mostrando um papel significante na área da saúde.

A RV tem sido amplamente utilizada na área da saúde como ferramenta de treinamento (Bigdeli \& Kaufman, 2017; Dörner et al., 2016; Malhotra et al., 2017; Wang et al., 2016). Estes sistemas computacionais utilizando RV podem ser aplicados na educação como ferramenta de aprendizado, por exemplo, ao utilizar jogos sérios. Isto permite ao usuário praticar um processo de exploração, descoberta e aprendizado. Ou seja, a RV pode proporcionar não somente a teoria, mas também experimentação prática (Cardoso et al., 2007). Nestes sistemas, aspectos como qualidade da imagem, campo de visão, vivacidade, interatividade, enredo, etc. podem ser usados para definir e caracterizar o tipo e nível de imersão (Tori \& da Silva Hounsell, 2020).

Ainda na via de RV, os jogos sérios (serious games) são jogos digitais que possuem um viés de treinamento, simulação, educação com propósitos específicos. Esta abordagem tem objetivo de unir conteúdos específicos e ludicidade, motivando o processo de aprendizado (Machado et al., 2011). Segundo a definição de (Dörner et al., 2016) os jogos sérios são 
jogos digitais criados com a intenção de entreter e alcançar um objetivo adicional, como aprendizado ou saúde.

Desta forma, com a crescente utilização da RV na área da saúde para treinamento, este trabalho tem o objetivo de realizar uma breve revisão bibliográfica, apresentando o estado da arte de sistemas de treinamento que utilizam a geração de imagem através de raios X. Assim, este trabalho apresenta e relaciona diferentes sistemas voltados para a área de radiologia aplicando técnicas de RV, assim como simuladores e jogos sérios.

\section{Metodologia}

De acordo com (Yin, 2015), o estudo de caso como estratégia de pesquisa aborda aspectos relacionados à coleta e análise de dados. Segundo esse autor, um estudo neste modelo pode ser de caso único ou de casos múltiplos. Desta forma, este trabalho segue a metodologia de pesquisa de estudos de casos múltiplos, apresentando a coleta e a análise qualitativa dos dados recolhidos do estado da arte de múltiplos trabalhos.

A busca de trabalhos foi realizada no idioma inglês utilizando as bases e bibliotecas digitais:

- ACM Digital Library;

- IEEE Xplore Digital Library;

- ScienceDirect;

- Web of Science;

- Wiley Online Library.

Foram utilizadas as seguintes palavras-chave: "medical training", "virtual environment", "serious game", "gamification", "image acquisition", “x-rays" e "mammography”. A frase de busca (string) foi criada a partir da combinação com das palavras-chave e adaptada para cada ferramenta de busca, com suas especificidades. A seguinte string de busca foi utilizada:

("virtual environment" OR "serious game" OR gamification) AND ((mammography OR "image acquisition" OR " $\mathrm{x}$ rays") AND (training OR education))

Durante a pesquisa foi aplicado o filtro para trabalhos publicados entre o ano de 2014 e 2021. Trabalhos que não abordavam treinamento na área da saúde e não incluíam ambientes virtuais com procedimentos médicos e não apresentavam alguma relação com aquisição de imagens médicas foram excluídos. A partir desta triagem, foi identificado um total de 337 trabalhos e a partir do resumo foram filtrados e selecionados 5 trabalhos mais relevantes para comparação nos seguintes aspectos:

Gamificação: Aplicação de técnicas de gamificação como pontuação por ações, feedback, implementação de níveis (fases), sistema de recompensa, bem como elementos sugerindo uma atividade lúdica.

Recursos educacionais customizáveis (modificações): É possível adicionar recursos educacionais ou adicionar/alterar tarefas no sistema. Ou seja, o sistema permite ou facilita a inserção ou alteração de novos conteúdos e/ou questionários através de uma interface ou formulário.

Registro de desempenho e geração de relatório (relatório): O sistema possui registro do desempenho do usuário e possibilita a geração de relatório de desempenho para avaliação ao longo do tempo o entre os questionários. Também foi observado se o sistema possui um mecanismo de seção individual de usuário (perfis).

Questionário de avaliação (avaliação): O sistema possui mecanismo próprio para dar a nota (score) para o usuário e gerar um sistema de pontuação em questionários. Foi observado se o sistema não necessita de um avaliador externo (como um profissional) para julgar se um procedimento executado está correto ou não.

Qualidade dos elementos gráficos (realismo): classificação dos elementos componentes gráficos do sistema. Foram observados aspectos dos modelos 3D como qualidade das texturas e quantidade de polígonos. Também foi observado a 
iluminação no ambiente em relação à presença e qualidade dos shaders (sombras, reflexos e Subsurface scattering (Nguyen, 2007)). Para uma avaliação mais precisa, seria necessário um teste com a aplicação produzida por cada autor e acesso aos objetos das cenas, o que seria inviável.

Tipo de equipamento (equipamento): Categoria de equipamento utilizado para treinamento no ambiente virtual.

Tabela 1. Estão relacionados os assuntos, as referências e o número de trabalhos qualitativos e quantitativos que foram usados nesse artigo.

\begin{tabular}{|c|c|c|c|c|c|c|}
\hline Trabalho & 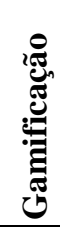 & 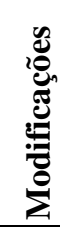 & 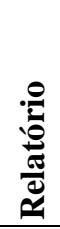 & 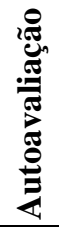 & 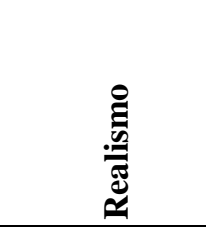 & 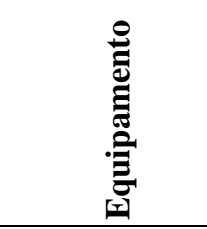 \\
\hline (Süncksen et al., 2018) & $\mathrm{X}$ & $\mathrm{X}$ & & $\mathrm{X}$ & Baixo/Médio & Arco C \\
\hline (Bridge et al., 2014) & & & & & Alto & Convencional \\
\hline (Wilcocks et al., 2018) & & & & & Médio & Arco C \\
\hline (O’Connor et al., 2021) & & & $\mathrm{X}$ & $\mathrm{X}$ & Alto & Convencional \\
\hline (Sapkaroski et al., 2018) & & & $\mathrm{X}$ & $\mathrm{X}$ & Médio & Convencional \\
\hline
\end{tabular}

Fonte: Autores.

\subsection{Gamification and virtual reality for teaching mobile $x$-ray imaging (Süncksen et al., 2018)}

Neste trabalho, (Süncksen et al., 2018) apresenta elementos de gamificação e RV para treinamento de um dispositivo de raios $\mathrm{X}$ do tipo arco $\mathrm{C}$. O sistema é voltado para o ensino e treinamento de operadores do equipamento em procedimentos de imagem intraoperativos.

Neste e em outros equipamentos de imagem para procedimentos intraoperativos, raios $\mathrm{X}$ são utilizados continuamente em boa parte dos procedimentos de cirurgia. Os autores citam a necessidade de educação e treinamento para minimizar a exposição inadequada e desnecessária de raios $\mathrm{X}$ ao paciente e profissionais durante as operações.

O sistema demonstrado neste trabalho foi construído com a engine Unity3D. O autor apresenta no ambiente virtual os elementos de uma sala de operações comum, contendo o equipamento de imagem arco C, mesa de operações, tela (para exibição da imagem de raios X) e um paciente. O sistema pode ser utilizado em modo desktop ou em modo Head-Mounted Dsisplay (HMD).

A partir da interface do sistema (mostrada na Figura 1), o usuário pode mover o paciente, posicionar e tirar imagens com o equipamento. As instruções para o usuário são exibidas na tela em forma de texto e imagem, com informações da estrutura anatômica alvo a ser capturada, ângulo de posicionamento do arco C. 
Figura 1. Captura de tela do sistema apresentado (Süncksen et al., 2018). A definição dos elementos (A, B, etc.) podem ser encontrados na publicação original.

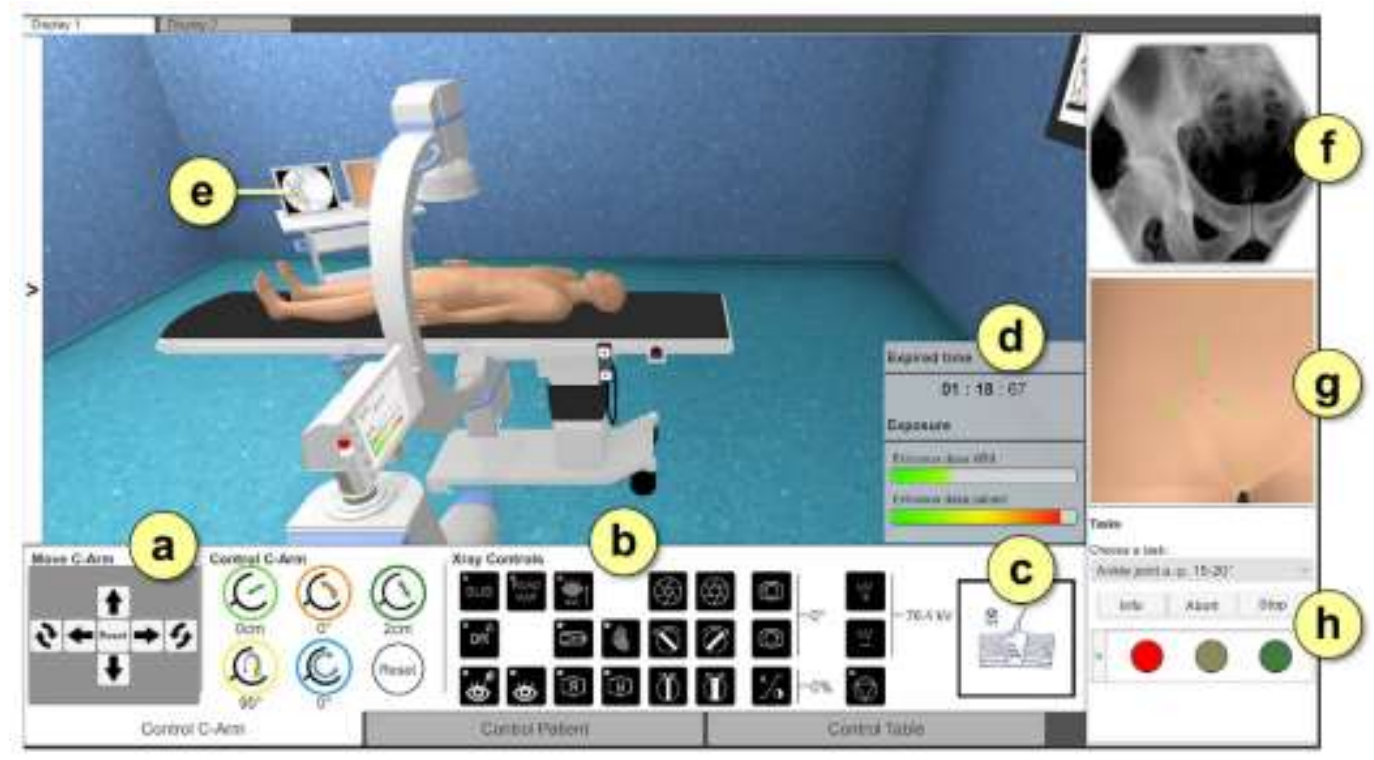

Fonte: Adaptado de (Süncksen et al., 2018).

As tarefas são avaliadas de acordo com critérios como exatidão do posicionamento do equipamento (ângulo da imagem), tempo de execução da tarefa e dose acumulada no paciente. O sistema permite ainda um modo de autoria, que permite adição ou alteração de tarefas por outro usuário.

$\mathrm{Na}$ avaliação de tarefas, uma tela é apresentada com os parâmetros atingidos pelo usuário, bem como instruções curtas com dicas para melhoria do procedimento. O autor afirma ainda que o objetivo principal do jogo é a realização de boas imagens radiográficas. Para elemento de gamificação, o jogo apresenta características como aumento de dificuldade nos níveis, desafios lúdicos e sistema de recompensas por pontos com base nos procedimentos executados.

O sistema apresenta como vantagem a capacidade de gerar imagens virtuais a partir de interações do usuário no sistema e possibilidade de modificação das tarefas por outro usuário experiente. Como desvantagem, o trabalho não menciona a geração de um histórico de pontuação e/ou desempenho (relatório) do usuário para avaliação posterior. Pode-se destacar também como desvantagem o realismo de qualidade baixa/intermediária.

\subsection{The development and evaluation of a medical imaging training immersive environment (Bridge et al., 2014)}

Em (Bridge et al., 2014), é destacado que para o treinamento e simulação de equipamentos radiográficos é necessário um ambiente completo, diferente daqueles para treinamento de cirurgias como broncoscopia e colocação de cateter. Os autores também citam que as soluções já existentes geralmente não proporcionam um bom nível de realismo e um nível interações adequadas.

O sistema Medical Imaging Training Immersive Environment (MITIE) de treinamento, tem o objetivo de prover um treinamento para estudantes de imagens médicas equivalentes ao recebido por estudantes em cursos de radiografia. O sistema permite experimentação e conta com feedback automatizado que destaca erros e melhorias possíveis nos procedimentos executados no ambiente virtual.

$\mathrm{O}$ ambiente foi criado por uma empresa especializada utilizando a engine Quest $3 D$. O sistema possui alto realismo gráfico dos equipamentos, sala e área de controle. Também possui um modelo com rig de paciente, permitindo posicionamento 
do mesmo e captura de imagens. O sistema é possível de ser utilizado em desktops ou com óculos do tipo shutter glasses. Uma pré-visualização pode ser observada na Figura 2.

Para avaliação do desempenho dos estudantes no sistema, foi considerado a habilidade de posicionar o paciente, o equipamento e reproduzir as projeções radiográficas. A avaliação das projeções radiográficas foi feita de forma independente por profissionais auxiliares.

Os resultados foram medidos a partir da aplicação de questionários e uma atividade avaliativa de performance com os estudantes divididos em 2 grupos. Um grupo recebeu treinamento prático e o outro recebeu treinamento pelo ambiente virtual. $\mathrm{Na}$ avaliação, o grupo que recebeu treinamento do ambiente virtual obteve melhor performance. No questionário, os estudantes mostraram preferência pela versão 2D do sistema.

Pode-se destacar como vantagem no sistema MITIE a alta fidelidade gráfica do ambiente de uma forma geral e a possibilidade de geração de imagens virtuais. Como desvantagem, o sistema não permite a adição ou modificação de tarefas e o artigo não menciona elementos gamificação como pontuação, diferentes níveis e sistema de recompensa.

Figura 2. Captura de tela do sistema MITIE.

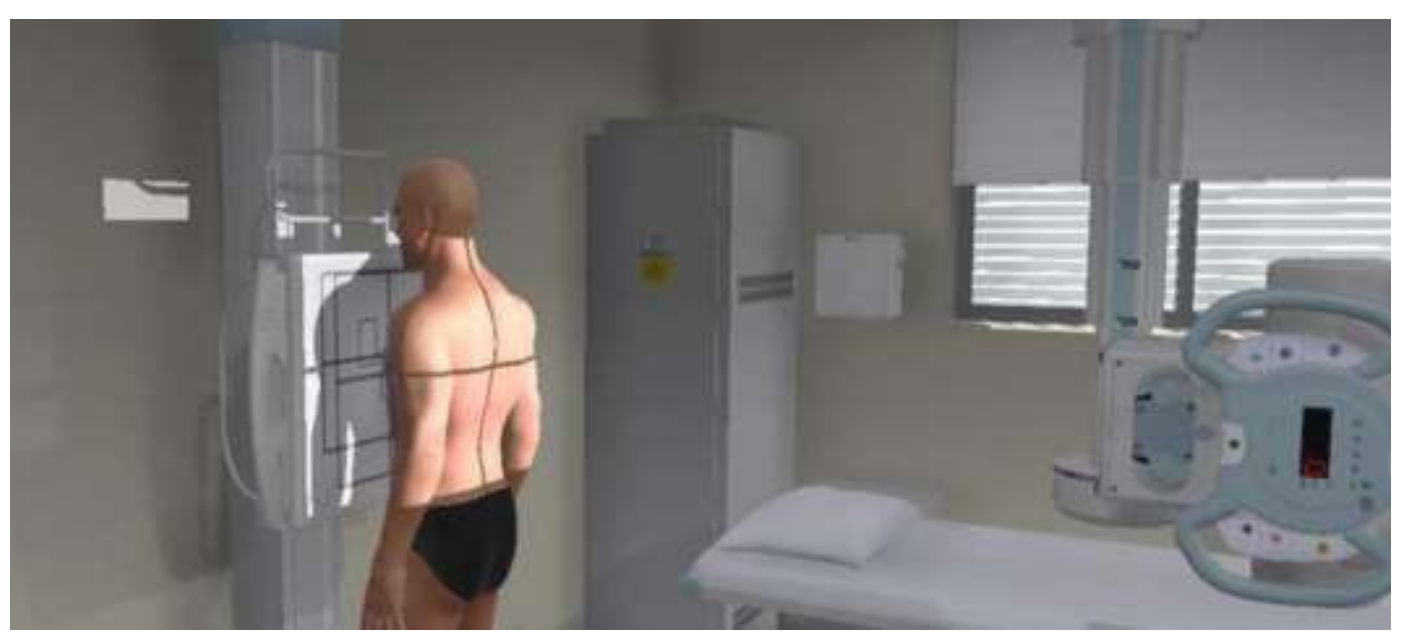

Fonte: Adaptado de (Bridge et al., 2014).

\subsection{A virtual cardiac catheterization laboratory for patient education: The angiogram procedure (Wilcocks et al., 2018)}

Neste trabalho (Wilcocks et al., 2018), é apresentado um simulador em um laboratório virtual de procedimentos de cateterização desenvolvido especificamente para educação e informação de pacientes. O sistema é focado em angiograma e emprega tecnologias imersivas para informar e educar pacientes submetidos ao procedimento.

O ambiente foi construído a partir da engine Unity3D para ser utilizado com óculos $H T C V_{i v e}{ }^{T M}$. Durante a simulação o paciente controla um avatar que pode navegar e interagir com elementos da cena. As instruções são fornecidas através de uma tela fixa com texto e um assistente virtual na própria cena que descreve de forma falada (narrada) o ambiente a guia as tarefas a serem executadas, como localização, posicionamento e aplicação das ferramentas. Uma pré-visualização do ambiente pode ser observada na Figura 3. 
Figura 3. Captura de tela do apresentado por (Wilcocks et al., 2018).

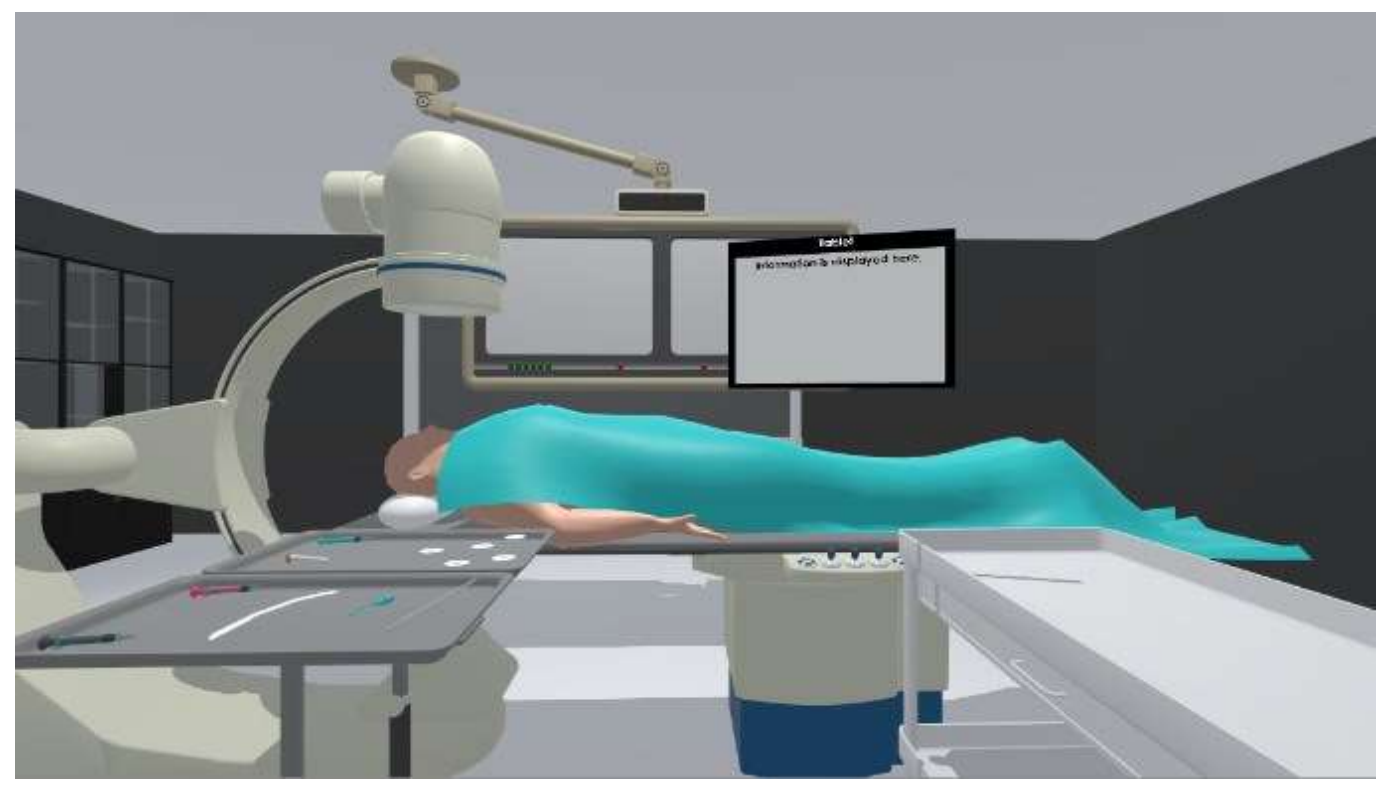

Fonte: Adaptado de (Wilcocks et al., 2018).

O realismo é médio para os equipamentos e sala virtual, em geral. $\mathrm{O}$ autor não cita elementos de gamificação. $\mathrm{O}$ trabalho também não cita a geração de relatório e avaliação interna (automática) do sistema. O equipamento apresentado no sistema é do tipo Arco C.

Como vantagem o sistema possui um mecanismo de apresentação de instruções através de fala (narração), que amplia a acessibilidade às pacientes com condições adversas ou deficiências. Como desvantagem, pode-se citar que o ambiente fornece apenas uma categoria de procedimento (angiograma), embora autor cite que no laboratório real diversos outros procedimentos são realizados. Também não é possível a adição de outras situações na cena. Não é citado a operação do equipamento no sistema.

\section{$2.43 D$ virtual reality simulation in radiography education: The students' experience (O'Connor et al., 2021)}

O trabalho de (O’Connor et al., 2021) avalia o impacto da adoção de um sistema em RV no treinamento piloto de estudantes do primeiro ano de graduação em Radiografia. O sistema em RV foi produzido pela empresa Virtual Medical Coaching Ltd, que atua no mercado de treinamento médico.

$\mathrm{O}$ trabalho tem o objetivo de determinar se com o uso do sistema os estudantes sentiram-se mais confiantes e teriam suas habilidades desenvolvidas, assim como identificar melhorias potenciais e investigar possível integração no currículo do curso de radiografia. Por fim, o estudo visa também avaliar as perspectivas dos estudantes acerca do uso da ferramenta em RV como método de avaliação.

A ferramenta utilizada neste trabalho, recorre aos óculos HTC VivePro ${ }^{\mathrm{TM}}$. As seções de treinamento com a ferramenta em RV com os estudantes foram divididas em módulos. O conteúdo é relacionado à projeção radiográfica de algumas partes do corpo (caixa torácica, pélvis, etc.). As simulações também abordavam conteúdo sobre anatomia, posicionamento radiográfico e patologias em cada parte do corpo. A Figura 4 exibe um usuário demonstrando a utilização do sistema. 
Figura 4. Captura de tela do sistema apresentado por (O’Connor et al., 2021).

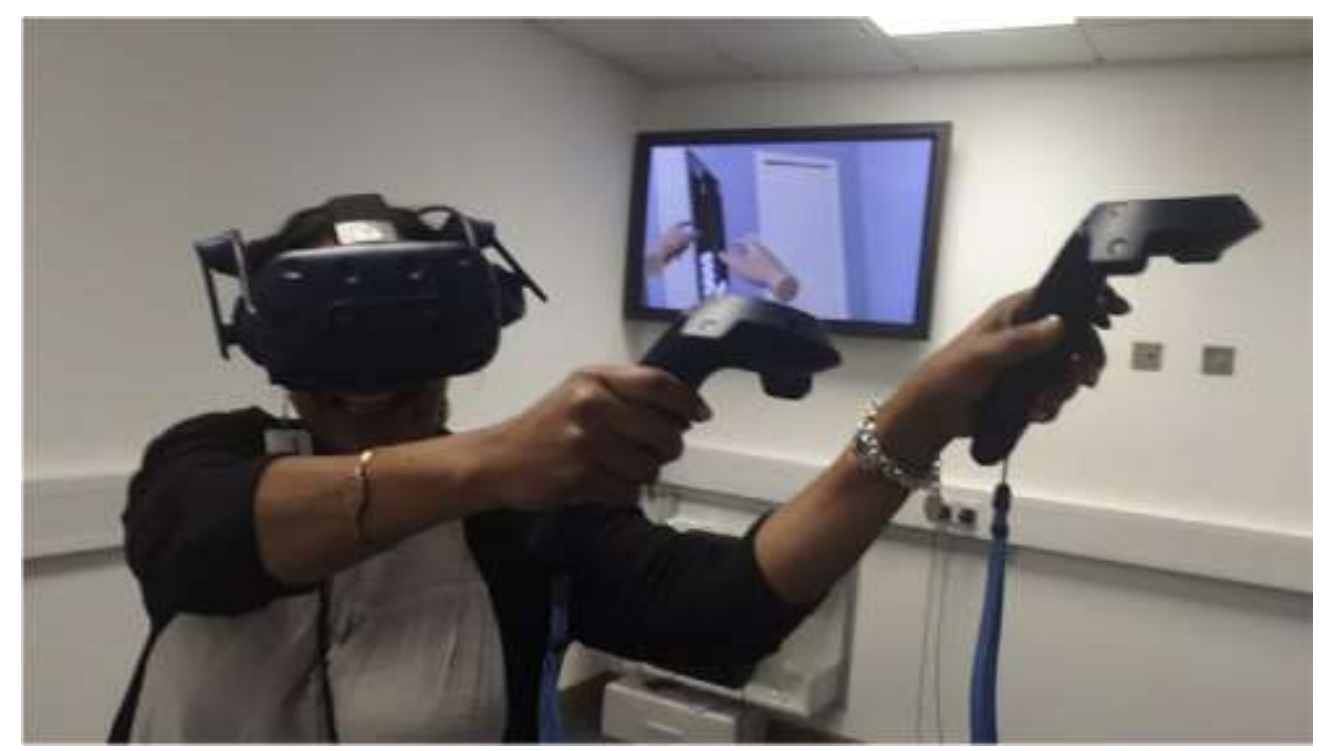

Fonte: Adaptado de (O'Connor et al., 2021).

Após responderem um questionário rápido (quiz) sobre conteúdo aprendido, um paciente virtual dentro de uma sala de operações de imagens era apresentado para que o usuário pudesse manipular o equipamento e tirar imagens raios $\mathrm{X}$ deste paciente virtual.

Ao finalizar as tarefas, um relatório é gerado contendo informações sobre a performance dos estudantes no quiz e simulador. No trabalho não foi abordado sistema de gamificação. Como conclusão, os autores puderam perceber que os estudantes se sentiram mais confiantes na aplicação das técnicas radiográficas após o uso da ferramenta e apreciaram a natureza interativa do simulador.

Como vantagem, pode-se destacar a qualidade das interações. Também pode-se mencionar a geração de relatório de desempenho pelo sistema, com feedback. Pela proposta do sistema, a falta de elementos de gamificação e pode ser considerada uma desvantagem. O sistema também não menciona possibilidade de alteração ou adição de tarefas.

\subsection{Virtual reality versus conventional clinical role-play for radiographic positioning training: A students' perception study} (Sapkaroski et al., 2018)

No trabalho de (Sapkaroski et al., 2018), foi criado o sistema Clinical Education Training Solution (CETSOL) para permitir a comunicação e interação com pacientes no ambiente bem como a aquisição e processamento de imagens. Este trabalho faz uma comparação com o software ShaderwareProjectionVR ${ }^{T M}$. O foi sistema desenvolvido durante 4 anos e adotado formalmente no currículo do curso de Bacharelado em Radiografia e Imagens Médicas na Universidade Monash, na Austrália.

O CETSOL foi desenvolvido com duas versões. Foi utilizada a engine Unity3D e conta com interações multimodais, podendo ser utilizado o Oculus Rift® ou HTC Vive ${ }^{\mathrm{TM}}$. As interações podem ser feitas utilizando o Leap Motion ${ }^{\circledR}$ também. O sistema utiliza uma Application Programming Interface (API) para reconhecimento de voz que permite uma comunicação dinâmica com o avatar do paciente além de possibilitar uma variabilidade de exames de imagem (caixa torácica, abdome, etc.). Um mecanismo de registro de progresso remoto dos estudantes também foi criado. A Figura 5 exibe uma pré-visualização do sistema. 
Figura 5. Captura de tela do sistema CETSOL apresentado por (Sapkaroski et al., 2018).

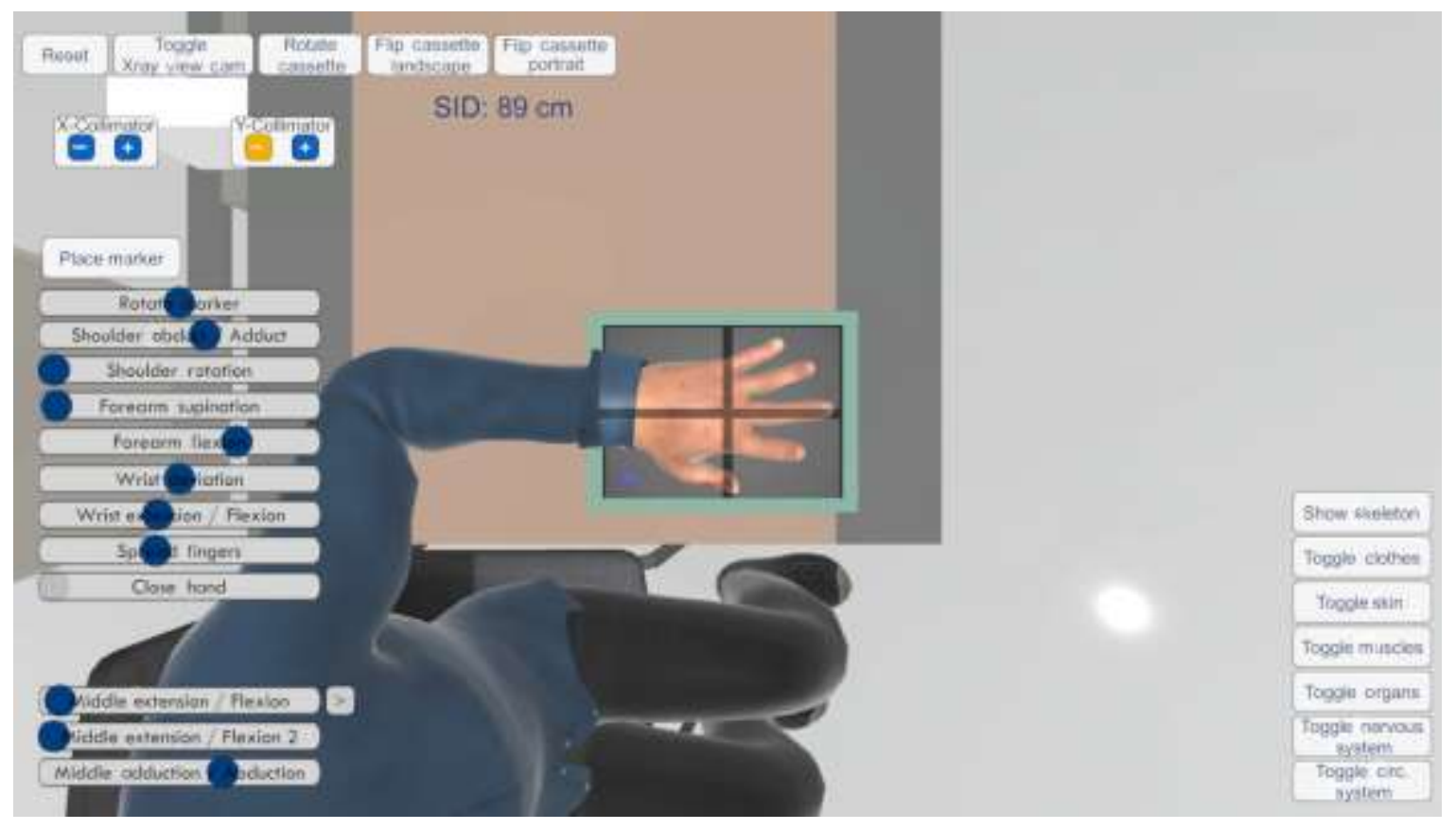

Fonte: Adaptado de (Sapkaroski et al., 2018).

No simulador, os estudantes foram instruídos a realizar uma tarefa de capturar uma imagem de mão completa. A tarefa envolvia a comunicação com o paciente virtual acerca do procedimento, seleção correta das ferramentas e configuração do equipamento (fatores como colimação, distância imagem-fonte e exposição).

Como vantagem do sistema, pode-se mencionar o alto nível de interatividade proporcionado, qualidade gráfica regular (médio), a possibilidade de uso individual e geração de relatórios para acompanhamento dos educadores. Como desvantagem, o sistema não permite adições ou modificações de conteúdo de ensino e o trabalho não cita a aplicação de elementos de gamificação. Avaliação e geração de relatórios não foram mencionados explicitamente no trabalho, porém o autor menciona a coleta de dados e implementação de estudo auto direcionado.

\section{Resultados e Discussão}

Os elementos de gamificação não se apresentam em todos os trabalhos, já que os mesmos apresentam uma proposta de simulador, com exceção do trabalho de (Süncksen et al., 2018). Sobre a possibilidade de modificação do sistema, novamente, apenas o trabalho de (Süncksen et al., 2018) mostra essa funcionalidade.

Sobre a capacidade de registro do desempenho dos usuários nos sistemas, pode-se observar que a funcionalidade se torna mais útil quando o sistema é utilizado em ambientes de ensino, pois permite que professores e educadores acompanhem a evolução dos estudantes. Apenas os estudos de (O’Connor et al., 2021; Sapkaroski et al., 2018) apresentam tais aspectos.

Em relação ao realismo, apenas os trabalhos de (Bridge et al., 2014) e (O’Connor et al., 2021) são os que possuem uma maior qualidade gráfica, apresentando alto realismo. O trabalho de (Sapkaroski et al., 2018; Wilcocks et al., 2018) exibe um realismo gráfico regular, assim, pode ser classificado com médio.

Em relação ao equipamento, os trabalhos encontrados utilizavam Arco $\mathrm{C}$ ou equipamento de raios $\mathrm{X}$ convencional. Não foram encontrados trabalhos com treinamento específico para a mamografia e/ou tomografia. Isto provavelmente deve-se aos altos níveis de realismo necessário para o desenvolvimento de aplicações completas. Por exemplo, na compressão de mamas é necessário a aplicação com física de corpos macios, que atualmente são complexas e custosas computacionalmente. 


\section{Conclusão}

Como mostrado nos trabalhos apresentados, o treinamento utilizando técnicas de RV são úteis no treinamento na área da saúde, apresentando diversas vantagens. Por exemplo, pode-se citar a possibilidade de utilização dos equipamentos virtuais sem risco de exposição real aos raios $\mathrm{X}$.

\section{Agradecimentos}

Os autores agradecem à CAPES (Coordenação de Aperfeiçoamento de Pessoal de Nível Superior) pelo auxílio financeiro dado a esta pesquisa.

\section{Referências}

Agência Nacional de Vigilância Sanitária. (2019). Resolução - RDC NO 330, de 20 de dezembro de 2019. Brasil, Ministério da Saúde. https://www.in.gov.br/web/dou/-/resolucao-rdc-n-330-de-20-de-dezembro-de-2019-235414748?inheritRedirect=true

Bigdeli, S., \& Kaufman, D. (2017). Digital games in medical education: Key terms, concepts, and definitions. Medical journal of the Islamic Republic of Iran, 31,52 .

Bridge, P., Gunn, T., Kastanis, L., Pack, D., Rowntree, P., Starkey, D., \& Wilson-Stewart, K. (2014). The development and evaluation of a medical imaging training immersive environment. Journal of medical radiation sciences, 61(3), 159-165.

Caldas, F. A. A., Isa, H. L. V. R., Trippia, A. C., Bíscaro, A. C. F. P. J., Souza, E. C. C., \& Tajara, L. M. (2005). Quality control and artifacts in mammography. Radiologia Brasileira, 38(4), 295-300.

Cardoso, A., Kirner, C., Júnior, E. L., \& Kelner, J. (2007). Tecnologias e ferramentas para o desenvolvimento de sistemas de realidade virtual e aumentada. Editora Universitária UFPE, 1-19.

Coop, P., Cowling, C., \& Lawson, C. (2016). Tomosynthesis as a screening tool for breast cancer: A systematic review. Radiography, $22(3)$, e190-e195.

Dörner, R., Göbel, S., Effelsberg, W., \& Wiemeyer, J. (2016). Serious games. Basel, Switzerland: Springer International Publishing.

INCA, I. N. de C. J. A. G. da S. (2015). Diretrizes para a detecção precoce do câncer de mama no Brasi. Serviço de Educação e Informação TécnicoCientífica Área de Edição e Produção de Materiais Técnico-Científicos.

INCA, I. N. de C. J. A. G. da S. (2019). Atualização em mamografia para técnicos em radiologia. Serviço de Educação e Informação Técnico-Científica Área de Edição e Produção de Materiais Técnico-Científicos.

INCA, I. N. de C. J. A. G. da S. (2021). Detecção precoce do câncer. Serviço de Educação e Informação Técnico-Científica Área de Edição e Produção de Materiais Técnico-Científicos.

Machado, L. D. S., Moraes, R. M. D., Nunes, F. D. L. D. S., \& Costa, R. M. E. M. D. (2011). Serious games baseados em realidade virtual para educação médica. Revista brasileira de educação médica, 35, 254-262.

Malhotra, V. M., Kabra, P. R., \& Malhotra, R. (2017). Attitudes and practices of medical students regarding video-games: should community medicine educationists get serious about serious games. International Journal Of Community Medicine And Public Health, 4(3), 729-733.

Migowski, A., Silva, G. A., Dias, M. B. K., Diz, M. D. P. E., Sant’Ana, D. R., \& Nadanovsky, P. (2018). Diretrizes para detecção precoce do câncer de mama no Brasil. II-Novas recomendações nacionais, principais evidências e controvérsias. Cadernos de Saúde Pública, 34 , e00074817.

Nguyen, H. (2007). GPU Gems 3 (3rd ed.). Addison-Wesley Professional. https://doi.org/10.5555/1536934

O'Connor, M., Stowe, J., Potocnik, J., Giannotti, N., Murphy, S., \& Rainford, L. (2021). 3D virtual reality simulation in radiography education: The students' experience. Radiography, 27(1), 208-214.

OPAS, O. P.-A. de S. (2020). Câncer, Fact Sheet. https://www.paho.org/en/topics/cancer.

Sabino, S. M. P. D. S., Silva, T. B., Watanabe, A. H. U., Syrjänen, K., Carvalho, A. L., \& Mauad, E. C. (2014). Implementation of a clinical quality control program in a mammography screening service of Brazil. Anticancer research, 34(9), 5057-5065.

Sapkaroski, D., Baird, M., McInerney, J., \& Dimmock, M. R. (2018). The implementation of a haptic feedback virtual reality simulation clinic with dynamic patient interaction and communication for medical imaging students. Journal of medical radiation sciences, 65(3), 218-225.

Süncksen, M., Bendig, H., Teistler, M., Wagner, M., Bott, O. J., \& Dresing, K. (2018, May). Gamification and virtual reality for teaching mobile x-ray imaging. In 2018 IEEE 6th International Conference on Serious Games and Applications for Health (SeGAH) (pp. 1-7). IEEE.

Tori, R., \& da Silva Hounsell, M. (2020). Introduçao a realidade virtual e aumentada. Interação, 7, 11.

Yin, R. K. (2015). Estudo de Caso: Planejamento e métodos. Bookman editora. 
Research, Society and Development, v. 10, n. 15, e55101522507, 2021

(CC BY 4.0) | ISSN 2525-3409 | DOI: http://dx.doi.org/10.33448/rsd-v10i15.22507

Wang, R., DeMaria Jr, S., Goldberg, A., \& Katz, D. (2016). A systematic review of serious games in training health care professionals. Simulation in Healthcare, 11(1), 41-51.

Wilcocks, K., Halabi, N., Kartick, P., Uribe-Quevedo, A., Chow, C., \& Kapralos, B. (2017, August). A virtual cardiac catheterization laboratory for patient education: The angiogram procedure. In 2017 8th International Conference on Information, Intelligence, Systems \& Applications (IISA) (pp. 1-4). IEEE. 